\title{
Niosomes as Nanoparticular Drug Carriers: Fundamentals and Recent Applications
}

\author{
Didem Ag Seleci, Muharrem Seleci, Johanna-Gabriela Walter, \\ Frank Stahl, and Thomas Scheper \\ Institute for Technical Chemistry, Leibniz University Hannover, 30167 Hannover, Germany \\ Correspondence should be addressed to Frank Stahl; stahl@iftc.uni-hannover.de
}

Received 21 April 2016; Accepted 23 May 2016

Academic Editor: Lei Liu

Copyright @ 2016 Didem Ag Seleci et al. This is an open access article distributed under the Creative Commons Attribution License, which permits unrestricted use, distribution, and reproduction in any medium, provided the original work is properly cited.

Drug delivery systems are defined as formulations aiming for transportation of a drug to the desired area of action within the body. The basic component of drug delivery systems is an appropriate carrier that protects the drug from rapid degradation or clearance and thereby enhances drug concentration in target tissues. Based on their biodegradable, biocompatible, and nonimmunogenic structure, niosomes are promising drug carriers that are formed by self-association of nonionic surfactants and cholesterol in an aqueous phase. In recent years, numerous research articles have been published in scientific journals reporting the potential of niosomes to serve as a carrier for the delivery of different types of drugs. The present review describes preparation methods, characterization techniques, and recent studies on niosomal drug delivery systems and also gives up to date information regarding recent applications of niosomes in drug delivery.

\section{Introduction}

Delivering drug with a controlled rate and targeted delivery received much attention in recent years. The application of nanotechnology to medicine has provided the development of multifunctional nanoparticles that, acting as drug carriers, can be loaded with different drugs. Nanocarriers present a great approach in drug delivery with promising features such as protection of drug from degradation and cleavage, controlled release, and in case of targeted delivery approaches the delivery of drug molecules to the target sites [1].

Niosomes are one of the promising drug carriers that have a bilayer structure and are formed by self-association of nonionic surfactants and cholesterol in an aqueous phase. Niosomes are biodegradable, biocompatible, and nonimmunogenic. They have long shelf life, exhibit high stability, and enable the delivery of drug at target site in a controlled and/or sustained manner [2]. In recent years, the potential of niosomes as a drug carrier has been extensively studied [35]. Various types of nonionic surfactants have been reported to form niosomes and enable the entrapment of a large number of drugs with a wide range of solubility [6-8]. The composition, size, number of lamellae, and surface charge of niosomes can be varied and optimized to enhance the performance of niosomes for drug delivery.

The aim of this review is to present the fundamentals of niosome preparation and characterization as well as a description of their use in drug delivery, with particular attention to more recent studies. This review will provide an overview on the increasing interest on niosomes in the field of drug delivery.

\section{Structure and Components of Niosomes}

The main components of niosomes are nonionic surfactants, hydration medium and lipids such as cholesterol. The list of materials used in the preparation of niosomes has been shown in Table 1 . The self-assembly of nonionic surfactants in aqueous media results in closed bilayer structures (Figure 1). A high interfacial tension between water and the hydrophobic tails of the amphiphile causes them to associate. The steric and hydrophilic repulsion between the head groups of nonionic surfactant ensure that hydrophilic termini point outwards and are in contact with water. The assembly into closed 
TABLE 1: The materials used in niosome preparation.

\begin{tabular}{|c|c|c|}
\hline Nonionic surfactants & Examples & References \\
\hline \multicolumn{3}{|l|}{ Alkyl ethers } \\
\hline (i) Alkyl glycerol ethers & Hexadecyl diglycerol ether (C16G2) & [9] \\
\hline (ii) Polyoxyethylene glycol alkyl ethers (Brij) & Brij 30, Brij 52, Brij 72, Brij 76, Brij 78 & {$[10-12]$} \\
\hline Crown ethers & Bola & {$[13,14]$} \\
\hline \multicolumn{3}{|l|}{ Alkyl esters } \\
\hline (i) Sorbitan fatty acid esters (Spans) & $\begin{array}{l}\text { Span 20, Span 40, Span 60, Span 80, Span 65, } \\
\text { Span } 85\end{array}$ & {$[15-18]$} \\
\hline (ii) Polyoxyethylene sorbitan fatty acid esters (Tweens) & $\begin{array}{l}\text { Tween 20, Tween 40, Tween 60, Tween 80, } \\
\text { Tween 65, Tween } 85\end{array}$ & {$[7,19,20]$} \\
\hline \multicolumn{3}{|l|}{ Alkyl amides } \\
\hline (i) Glycosides & C-Glycoside derivative surfactant (BRM-BG) & {$[21]$} \\
\hline (ii) Alkyl polyglucosides & $\begin{array}{l}\text { Octyl-decyl polyglucoside (OrCG110), decyl } \\
\text { polyglucoside (OrNS10) }\end{array}$ & {$[22]$} \\
\hline \multicolumn{3}{|l|}{ Fatty alcohols or fatty acids } \\
\hline (i) Fatty alcohols & Stearyl alcohol, cetyl alcohol, myristyl alcohol & [23] \\
\hline (ii) Fatty acids & Stearic acid, palmitic acid, myristic acid & [23] \\
\hline \multicolumn{3}{|l|}{ Block copolymer } \\
\hline (i) Pluronic & Pluronic L64, Pluronic 105 & {$[24,25]$} \\
\hline \multicolumn{3}{|l|}{ Lipidic components } \\
\hline Cholesterol & & {$[26]$} \\
\hline l- $\alpha$-Soya phosphatidyl choline & & {$[27]$} \\
\hline \multicolumn{3}{|l|}{ Charged molecule } \\
\hline Negative charge & $\begin{array}{l}\text { Diacetyl phosphate, phosphatidic acid, } \\
\text { lipoamino acid, dihexadecyl phosphate }\end{array}$ & {$[28,29]$} \\
\hline Positive charge & $\begin{array}{l}\text { Stearylamine, stearyl pyridinium chloride, } \\
\text { cetyl pyridinium chloride }\end{array}$ & {$[29]$} \\
\hline
\end{tabular}

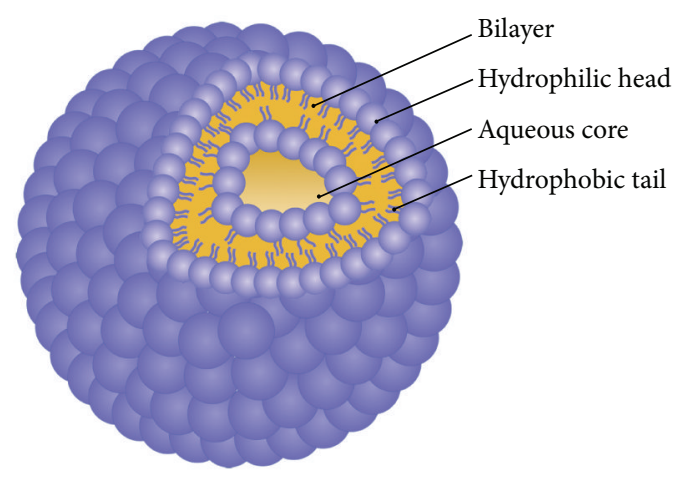

Figure 1: Structure of niosomes.

bilayers usually requires some input of energy such as mechanical or heat. Niosomes can be categorized in three groups according to their sizes and bilayers. Small unilamellar vesicles (SUV) (10-100 nm), large unilamellar vesicles (LUV) (100-3000 nm), and multilamellar vesicles (MLV) where more than one bilayer is present.

2.1. Nonionic Surfactants. Nonionic surfactants are a class of surfactants, which have no charged groups in their hydrophilic heads. They are more stable and biocompatible and less toxic compared to their anionic, amphoteric, or cationic counterparts [41]. Therefore they are preferred for formation of stable niosome for in vitro and in vivo applications. Nonionic surfactants are amphiphilic molecules that comprise two different regions: one of them is hydrophilic (water-soluble) and the other one is hydrophobic (organicsoluble). Alkyl ethers, alkyl esters, alkyl amides, fatty acids are the main nonionic surfactant classes used for niosome production. The hydrophilic-lipophilic balance (HLB) and critical packing parameter (CPP) values play a critical role in the selection of surfactant molecules for niosome preparation.

2.1.1. Hydrophilic-Lipophilic Balance (HLB). HLB is a dimensionless parameter, which is the indication of the solubility of the surfactant molecule. The HLB value describes the balance between the hydrophilic portion to the lipophilic portion of the nonionic surfactant. The HLB range is from 0 to 20 for nonionic surfactants. The lower HLB refers to more lipophilic surfactant and the higher HLB to more hydrophilic surfactant. Surfactants with a HLB between 4 and 8 can be used for preparation of vesicle [42]. Hydrophilic surfactants with a HLB value ranging from 14 to 17 are not suitable to form a bilayer membrane due to their high aqueous solubility [43]. 
However with the addition of an optimum level of cholesterol, niosomes are indeed formed from polysorbate 80 (HLB value $=15)$ and Tween $20($ HLB value $=16.7)[44,45]$. Tween 20 forms stable niosome in the presence of equimolar cholesterol concentration. The interaction occurs between the hydrophobic part of the amphiphile next to head group and the $3-\mathrm{OH}$ group of cholesterol at an equimolar ratio and this interaction could explain the effect of cholesterol on the formation and hydration behavior of Tween 20 niosomal membranes [46, 47].

Drug entrapment efficiency of the niosomes is also affected by HLB value of surfactant [48]. Shahiwala et al. have incorporated nimesulide into niosomes using lipid film hydration technique by changing the HLB. They found that as the HLB value of surfactant decreases from 8.6 to 1.7, entrapment efficiency decreases $[43,49]$.

2.1.2. Critical Packing Parameter (CPP). During the niosomal preparation, the geometry of the vesicle depends upon the critical packing parameter. On the basis of the CPP of a surfactant, the shape of nanostructures formed by self-assembly of amphiphilic molecules can be predicted. Critical packing parameter depends on the symmetry of the surfactant and can be defined using following equation $[50,51]$ :

$$
\mathrm{CPP}=\frac{v}{l_{c} \times a_{0}},
$$

where $v$ is hydrophobic group volume, $l_{c}$ is the critical hydrophobic group length, and $a_{0}$ is the area of hydrophilic head group. If CPP $\leq 1 / 3$ corresponding, for example, to a bulky head group, small hydrophobic tail spherical micelles may form. Nonspherical micelles may form if $1 / 3 \leq \mathrm{CPP} \leq$ $1 / 2$, and bilayer vesicles can occur if $1 / 2 \leq \mathrm{CPP} \leq 1$. Inverted micelles form if CPP $\geq 1$ when the surfactant is composed of a voluminous tail and a small hydrophobic tail [47]. CPP could be considered as a tool for realizing, rationalizing, and predicting the self-assembled structure and its morphological transition in amphiphilic solutions [52].

2.2. Cholesterol. In the bilayer structure of niosomes, cholesterol forms hydrogen bonds with hydrophilic head of a surfactant $[19,53]$. Cholesterol content of niosomes thereby influences the structures of niosomes and physical properties such as entrapment efficiency, long time stability, release of payload, and biostability $[17,46]$. Cholesterol improves the rigidity of vesicles and stabilizes niosomes towards destabilizing effects induced by plasma and serum components and decreases the permeability of vesicles for entrapped molecules thus inhibiting leakage [54].

Drug entrapment efficiency plays an important role in niosomal formulations and it can be altered by varying the content of cholesterol. Agarwal et al. demonstrated that cholesterol improves the stability of enoxacin loaded niosome with increasing cholesterol content, resulting in increases of entrapment efficiency [55]. The effect of cholesterol on flurbiprofen entrapment was studied by Mokhtar et al. and cholesterol was found to have little effect on the flurbiprofen entrapment into Span 20 and Span 80 niosomes. However, a significant increase in entrapment efficiency of flurbiprofen was obtained when $10 \%$ of cholesterol was incorporated into niosomes prepared from Span 40 and Span 60 followed by a decrease in encapsulation efficiency of the drug upon further increase in cholesterol content [56]. According to the reported results, the addition of cholesterol and its amounts needs to be optimized depending on the physical-chemical characteristic of surfactants and loaded drugs.

2.3. Charged Molecule. Charged molecules increase the stability of the vesicles by the addition of charged groups to the bilayer of vesicles. They increase surface charge density and thereby prevent vesicles aggregation. Dicetyl phosphate and phosphatidic acid are most used negatively charged molecules for niosome preparation and, similarly, stearylamine and stearyl pyridinium chloride are well-known positively charged molecules used in niosomal preparations. Normally, the charged molecule is added in niosomal formulation in an amount of $2.5-5 \mathrm{~mol} \%$. However increasing the amount of charged molecules can inhibit niosome formation [29].

\section{Methods of Preparation}

3.1. Thin-Film Hydration Method (TFH). Thin-film hydration method is a simple and well-known preparation method. In this method, the surfactants, cholesterol, and some additives such as charged molecules are dissolved in an organic solvent in a round bottomed flask. Then the organic solvent is removed using a rotary vacuum evaporator to obtain thin film on the inside wall of the flask. An aqueous solution of drug is added and the dry film is hydrated above the transition temperature $\left(T_{c}\right)$ of the surfactant for specified time with constant shaking $[57,58]$. Multilamellar niosomes are formed by this method.

3.2. Ether Injection Method (EIM). In ether injection method, the surfactants with additives are dissolved in diethyl ether and injected slowly through a needle in an aqueous drug solution maintained at a constant temperature, which is above the boiling point of the organic solvent. The organic solvent is evaporated using a rotary evaporator. During the vaporization the formation of single layered vesicles occurs [59-61].

3.3. Reverse Phase Evaporation Method (REV). In this method, niosomal ingredients are dissolved in a mixture of ether and chloroform and added to aqueous phase containing the drug. The resulting mixture is sonicated in order to form an emulsion and the organic phase is evaporated. Large unilamellar vesicles are formed during the evaporation of the organic solvent [62-64].

3.4. Microfluidization Method. The microfluidization method is based on submerged jet principle. In this method, the drug and the surfactant fluidized streams interact at ultrahigh velocities, in precisely defined micro channels within the interaction chamber. The high speed impingement and the energy involved leads to formation of niosomes. This method 
offers greater uniformity, smaller size, unilamellar vesicles, and high reproducibility in the formulation of niosomes [65, 66].

3.5. Supercritical Carbon Dioxide Fluid $\left(\mathrm{scCO}_{2}\right)$. Manosroi et al. have described the supercritical reverse phase evaporation technique for niosome formation $[67,68]$. They added Tween 61, cholesterol, glucose, PBS, and ethanol into the view cell and the $\mathrm{CO}_{2}$ gas was introduced into the view cell. After magnetic stirring until equilibrium, the pressure was released and niosomal dispersions were obtained [67]. This method enables one step production and easy scale-up.

3.6. Proniosome. Proniosome technique includes the coating of a water-soluble carrier such as sorbitol and mannitol with surfactant. The coating process results in the formation of a dry formulation. This preparation is termed "Proniosomes" which requires to be hydrated before being used. The niosomes are formed by the addition of the aqueous phase. This method helps in reducing physical stability problems such as the aggregation, leaking, and fusion problem and provides convenience in dosing, distribution, transportation, and storage showing improved results compared to conventional niosomes [69].

3.7. Transmembrane $p H$ Gradient. In this method, surfactant and cholesterol are dissolved in chloroform and evaporated to form a thin lipid film on the wall of a round bottomed flask. The film is hydrated with a solution of citric acid $(\mathrm{pH}=4)$ by vortex mixing and the resulting product is freeze-thawed for niosome formation. The aqueous solution of drug is added to this niosomal suspension, after that phosphate buffer is added to maintain $\mathrm{pH}$ between 7.0 and 7.2 [70]. According to this method, the interior of niosome has a more acidic $\mathrm{pH}$ value than the outer medium. The added unionized drug passes through the niosome membrane and enters into the niosome. The drug ionizes in an acidic medium and cannot escape from the niosomal bilayer [71].

3.8. Heating Method. This is a patented method which was created by Mozafari et al. [72, 73]. Surfactants and cholesterol are separately hydrated in buffer and the solution is heated to $120^{\circ} \mathrm{C}$ with stirring to dissolve cholesterol. The temperature is reduced and surfactants and other additives are then added to the buffer in which cholesterol is dissolved while stirring continues. Niosomes form at this stage, are left at room temperature, and then are kept at $4-5^{\circ} \mathrm{C}$ under nitrogen atmosphere until use [53].

3.9. The "Bubble" Method. In this method, surfactants, additives, and the buffer are added into a glass flask with three necks. Niosome components are dispersed at $70^{\circ} \mathrm{C}$ and the dispersion is mixed with homogenizer. After that, immediately the flask is placed in a water bath followed by the bubbling of nitrogen gas at $70^{\circ} \mathrm{C}$. Nitrogen gas is passed through a sample of homogenized surfactants resulting in formation of large unilamellar vesicles [74].

\section{Characterization of Niosomes}

The characterization of niosome is essential for the clinical applications. Characterization parameters have a direct impact on the stability of niosomes and a significant effect on their in vivo performance. Therefore these parameters such as morphology, size, polydispersity index (PI), number of lamellae, zeta potential, encapsulation efficiency, and stability must be evaluated.

4.1. Size and Morphology. Dynamic light scattering (DLS) [75], scanning electron microscopy (SEM) [76], transmission electron microscopy (TEM) [77], freeze fracture replicationelectron microscopy (FF-TEM) [68], and cryotransmission electron microscopy (cryo-TEM) [67] are the most used methods for the determination of niosome sizes andmorphology. DLS provides simultaneously cumulative information of particle size and valuable information on the homogeneity of the solution. A single sharp peak in the DLS profile implies existence of a single population of scatterers. The PI is helpful in this respect. It less than 0.3 corresponds to a homogenous population for colloidal systems [75]. The microscopic approaches are generally used to characterize the morphology of the niosomes.

4.2. Zeta Potential. Surface zeta potential of niosomes can be determined using zetasizer and DLS instruments. The surface charge of niosome plays an important role in the behavior of niosomes. In general, charged niosomes are more stable against aggregation than uncharged vesicles. Bayindir and Yuksel prepared paclitaxel loaded niosomes and investigated the physicochemical properties such as zeta potential of niosomes. They found that negative zeta potential values ranging between -41.7 and $-58.4 \mathrm{mV}$ are sufficiently high for electrostatic stabilization of niosomes [12].

4.3. Bilayer Characterization. Bilayer characteristics of niosomes have an importance on drug entrapment efficiency. The number of lamellae can be determined by AFM, NMR, and small angle X-ray scattering (SAXS) for multilamellar vesicles [54]. Membrane rigidity of niosomal formulations can be measured by means of the mobility of fluorescence probe as a function of temperature [20]. DPH (1,6 diphenyl$1,3,5$-hexatriene) is most used fluorescent probe and added to niosomal dispersion. DPH normally exists in hydrophobic region in the bilayer membrane. The microviscosity of niosomal membrane is determined by fluorescence polarization. High fluorescence polarization means high microviscosity of the membrane [78]. Moreover, the bilayer thickness can be characterized using the latter method, together with the in situ energy-dispersive X-ray diffraction (EDXD) [79].

4.4. Entrapment Efficiency. Entrapment efficiency (EE\%) is defined as the portion of the applied drug which is entrapped by the niosomes. Unencapsulated free drug can be removed from the niosomal solution using centrifugation [80], dialysis [24], or gel chromatography [81]. After this step the loaded drug can be released from niosomes by destruction of 




Figure 2: Niosomes in drug delivery.

vesicles. Niosomes can be destroyed with the addition of $0.1 \%$ Triton X-100 or methanol to niosomal suspension. The loaded and free drug concentration can be determined by a spectrophotometer [82] or high-performance liquid chromatography (HPLC) [83].

4.5. Stability. The stability of niosomes can be evaluated by determining mean vesicle size, size distribution, and entrapment efficiency over several month storage periods at different temperatures. During storage the niosomes are sampled at regular intervals of time and the percentage of drug which is retained into the niosomes is analyzed by UV spectroscopy or HPLC methods [82, 84].

4.6. In Vitro Release. One often applied method to study in vitro release is based on using of dialysis tubing. A dialysis bag is washed and soaked in distilled water. After 30 mins, the drug loaded niosomal suspension is transferred, into this bag. The bag containing the vesicles is immersed in buffer solution with constant shaking at $25^{\circ} \mathrm{C}$ or $37^{\circ} \mathrm{C}$. At specific time intervals, samples were removed from the outer buffer (release medium) and replaced with the same volume of fresh buffer. The samples are analyzed for the drug content by an appropriate assay method [17].

\section{Niosomes as Drug Carriers}

Niosomes are very promising carriers for the delivery of numerous pharmacological and diagnostic agents. A number of publications have reported the preparation, characterization, and use of niosomes as drug carriers. Because of their nonionic nature, they offer excellent biocompatibility and low toxicity. The unique structure of niosomes allows the development of effective novel drug delivery systems with ability of loading both hydrophilic and lipophilic drugs. Hydrophilic drugs and lipophilic drugs are entrapped into the aqueous core and membrane bilayer of niosome respectively (Figure 2).

5.1. Anticancer Drug Delivery. The current treatment for cancer is usually chemotherapy. The therapeutic efficacy of many anticancer drugs is limited by their poor penetration into tumor tissue and by their severe side effects on healthy cells. Various attempts have been made to overcome these drawbacks, including the use of niosomes as a novel drug delivery system.

5.1.1. Melanoma. Artemisone is a 10-amino-artemisinin derivative exhibiting antimalarial activity and also possessing antitumor activity. Dwivedi et al. encapsulated artemisone in niosomes using thin-film hydration method. The formulations showed highly selective cytotoxicity towards the melanoma cells with negligible toxicity towards the normal skin cells [85]. 5-Fluorouracil (5-FU), largely used in the treatment of different forms of skin cancers, was encapsulated in an innovative bola-niosomal system made up of $\alpha, \omega$-hexadecyl-bis-(1-aza-18-crown-6) (bola-surfactant), Span 80, and cholesterol. The percutaneous permeation of 5-FU-loaded bola-niosomes was evaluated by using human stratum 
corneum and epidermis membranes. Bola-niosomes provided an increase of the drug penetration of 8 - and 4-fold with respect to free drug aqueous solution [13]. The use of cisplatin is limited due to its severe toxic effects. Gude et al. synthesized niosomal cisplatin by using Span 60 and cholesterol and investigated the antimetastatic activity in experimental metastatic model of B16F10 melanoma. Their results suggest that cisplatin encapsulated in niosomes has significant antimetastatic activity and reduced toxicity when compared to free cisplatin [86].

\subsubsection{Breast Cancer. 5-FU-loaded polyethylene glycol- (PEG-)} coated and uncoated bola-niosomes were prepared by Cosco et al. and were tested on breast cancer cell lines (MCF7 and T47D). Both bola-niosome formulations provided an increase in the cytotoxic effect with respect to the free drug. In vivo experiments on MCF-7 xenograft tumor SCID mice models showed a more effective antitumor activity of the PEGylated niosomal 5-FU at a concentration ten times lower $(8 \mathrm{mg} / \mathrm{kg})$ than that of the free solution of the drug $(80 \mathrm{mg} / \mathrm{kg})$ after a treatment of 30 days [87]. Cantharidinentrapped niosomes were prepared by injection method. Their potential in enhancing the antitumor activities of the drug and reducing its toxicity was evaluated on human breast cancer cell line MCF-7. Moreover, in vivo therapeutic efficacy was investigated in $\mathrm{S}_{180}$ tumor-bearing mice. Mice treated with $1.0 \mathrm{mg} / \mathrm{kg}$ niosomal cantharidin showed the most effective antitumor activity, with an inhibition rate of $52.76 \%$, which was significantly higher than that of the same concentration of free cantharidin (1.0 mg/kg, 31.05\%) [88]. Recently, tamoxifen citrate niosomes were prepared by film hydration technique for localized cancer therapy through in vitro breast cancer cytotoxicity as well as in vivo solid antitumor efficacy. The optimized niosomal formulation of tamoxifen showed significantly enhanced cellular uptake (2.8-fold) and exhibited significantly greater cytotoxic activity on MCF-7 breast cancer cell line. In vivo experiments showed enhanced tumor volume reduction induced by niosomal tamoxifen when compared to free tamoxifen [89].

5.1.3. Ovarian Cancer. Uchegbu et al. prepared doxorubicin loaded niosomes. The activity of doxorubicin in hexadecyl diglycerol ether $\left(\mathrm{C}_{16} \mathrm{G}_{2}\right)$ and Span 60 niosomes was studied against a human ovarian cancer cell line and its doxorubicin resistant subline. According to the results, there was a slight reduction in the $\mathrm{IC}_{50}$ against the resistant cell line when the drug was encapsulated in Span 60 niosomes in comparison to the free drug in solution [90].

5.1.4. Lung Cancer. Adriamycin was encapsulated into the noisome using a monoalkyl triglycerol ether by Kerr et al. and the activity of niosomal adriamycin compared with free adriamycin solution on human lung tumor cells grown in monolayer and spheroid culture and in tumor xenografted nude mice. The growth delay (i.e., the time taken for the tumor volume to double) was significantly longer for adriamycin (15 days) and niosomal adriamycin (11 days) than for control (5.8 days). It is possible that the therapeutic ratio of adriamycin could be further enhanced by administration in niosomal form [91]. In another study, pentoxifylline loaded niosomes were prepared by lipid film hydration method. Intravenous administration of niosomal pentoxifylline $(6 \mathrm{mg} / \mathrm{kg}$ and $10 \mathrm{mg} / \mathrm{kg}$ ) resulted in significant reduction in lung nodules in an experimental metastatic B16F10 model suggesting accumulation of pentoxifylline in a distant target. Light microscopic observation of histologic sections showed a decrease in number of tumor islands in the lung [92].

5.2. Targeted Delivery. The efficiency and particularly the specificity of cellular targeting of niosomal drug delivery systems can be further improved by active targeting for tumor therapy, by using a ligand coupled to the surface of niosomes, which could be actively taken up, for example, via a receptor-mediated endocytosis. Niosome surfaces can be conjugated with small molecules and/or macromolecular targeting ligands to enable cell specific targeting [93]. Proteins and peptides, carbohydrates, aptamers, antibodies, and antibody fragments are the most commonly used molecules that bind specifically to an overexpressed target on the cell surface [94-96]. Bragagni et al. developed brain targeted niosomal formulation using with the glucosederivative as a targeting ligand. They formulated niosomal doxorubicin composed of span : cholesterol : solulan : Npalmitoylglucosamine. Preliminary in vivo studies in rats showed that intravenous administration of a single dose of the developed targeted-niosomal formulation with respect to the commercial one was able to significantly reduce the hearth accumulation of the drug and to keep it longer in the blood circulation and also to allow the achievement of well detectable doxorubicin brain concentrations [30]. Moreover, an efficient tumor-targeted niosomal delivery system was designed by Tavano et al. Niosomes were prepared from a mixture of Pluronic L64 surfactant and cholesterol and doxorubicin was entrapped into the niosome. After the preparation, transferrin was conjugated to niosomes surface using EDC (N-[3-(dimethylamino)propyl]-Nethylcarbodiimide hydrochloride) chemistry. Doxorubicinloaded niosome anticancer activity was achieved against MCF-7 and MDA-MB-231 tumor cell lines, and a significant reduction in viability in a dose and time related manner was observed [24]. The information about some recent studies on niosomal targeted drug delivery is summarized in Table 2.

5.3. Codrug Delivery. In recent years, nanoparticles have emerged as a promising class of carriers in codelivery of multiple drugs for combination therapy [97]. Combinational therapies enhance therapeutic efficacy and decrease dosage while obtaining equal or greater levels of efficacy and reducing drug resistance [98]. Anticancer drugs often have serious side effects. With multidrug delivery system Pasut et al. achieved higher anticancer activity for carcinoma cells, whereas multidrug delivery system decreased cytotoxicity against endothelial cells and cardiomyocytes, with respect to free drug treatment. In their system, they have developed simultaneous anticancer drug epirubicin and nitric oxide carrying system, in which nitric oxide and epirubicin were covalently conjugated to each terminal of PEG. Nitric oxide acts 


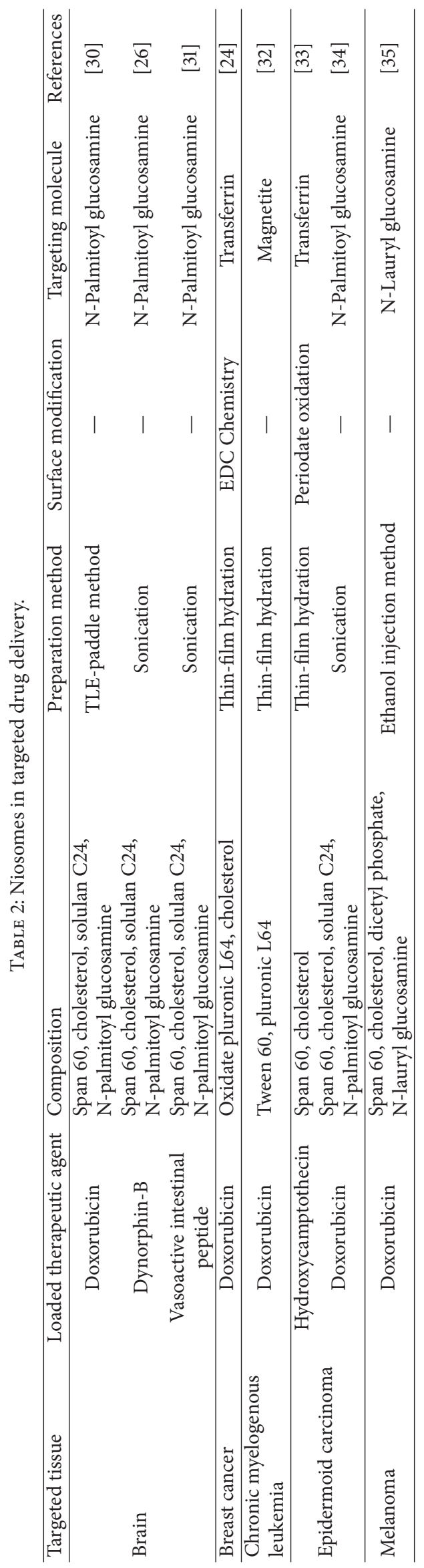


as not only protecting reagent against anthracycline induced cardiomyopathy but also sensitizer of anticancer drug treatment. In order to increase anticancer efficacy and enhance cardiocyte protecting ability of codelivery system, they used branched PEG as polymer backbone instead of linear one [99]. Multidrug resistance (MDR) of malignant neoplasm is the survival ability of cancer cells under the treatment with structurally and functionally diverse anticancer drugs. Increased drug efflux is mostly mediated by ATP-driven extrusion pump proteins of the ATP-binding cassette (ABC) superfamily, such as P-glycoprotein (P-gp) encoded by MDR1 , multidrug resistance (MDR) proteins (MRPs/ABCC) and breast cancer resistance protein (BCRP/ABCG2). These drug efflux pumps noticeably decrease the intracellular concentration of numerous therapeutic agents [100]. Chemosensitizers, such as Verapamil, Elacridar, Tariquidar, and cyclosporine A mainly act as antagonist for P-gp and suppress drug efflux and consequently recover chemosensitivity of MDR cancer cells. Paclitaxel was coencapsulated with cyclosporine A within actively targeted polymeric lipid-core micelles. P-gp inhibition with cyclosporine A caused an enhanced cytotoxicity of paclitaxel. Micelles loaded with this dual cargo demonstrated significantly higher cytotoxicity in the MDCKII-MDR1 cells than micelles loaded with paclitaxel alone [101].

Niosomes are promising nanocarriers in multidrug delivery applications [102]. Recently Sharma et al. reported the dual encapsulation of hydrophobic curcumin and hydrophilic doxorubicin in niosomes for cancer multidrug delivery [44]. Results showed that dual-drug loaded niosomes had higher cytotoxicity on HeLa cells when compared to free drugs. In another study, gallic acid, ascorbic acid, curcumin, and quercetin were encapsulated into the niosome as single agents or in combination and the effect of the drugs coencapsulation on the physicochemical properties of the carriers, on their antioxidant properties and capability to release the encapsulated materials, was evaluated [103]. Furthermore, Marianecci et al. prepared, characterized, and applied multidrug niosomes using lidocaine and ibuprofen. Results suggest the potential application of niosomes in dermal administration of the two drugs at the same time in the same pharmaceutical formulation, as useful carriers for the treatment of various skin diseases, such as acute and chronic inflammations in presence of pain [104].

5.4. Antibiotics. Niosomal carriers are also suitable for the delivery of antibiotics and anti-inflammatory agents. These carriers have been used extensively to improve poor skin penetration and as well as enhance skin retention of the drugs. Begum and coworkers designed rifampicin, a broad spectrum antibiotic, encapsulated in a niosomal delivery system. They investigated the activity of this system in in vitro conditions and this study showed that niosomal formulation of rifampicin is able to provide consistent and prolonged release of the drug [105]. In another study to increase efficacy of the antibiotics and reduce the dose, Akbari et al. synthesized ciprofloxacin loaded niosomes using different nonionic surfactants and cholesterol in various concentrations by film hydration method. Drug release through bilayers and antibacterial activity of the niosomes were examined.
The results showed that cholesterol content and phase transition temperature of the surfactants influenced the performance of niosomes. Besides, all formulations presented more antibacterial activity as compared to free ciprofloxacin [106].

Vesicular systems, niosomes and liposomes, are mostly used in ophthalmic controlled delivery. Abdelbary and ElGendy examined the feasibility of the niosomes as a carrier for the ophthalmic controlled delivery of gentamicin antibiotic. Various surfactants (Tween 60, Tween 80, or Brij 35) were combined with cholesterol and a negative charge inducer dicetyl phosphate in different molar ratios. The ability of these vesicles to entrap the selected drug was evaluated and the obtained results showed that entrapment efficiency and the release rate of gentamicin is affected by cholesterol content, type of surfactant, and the presence of charge inducer. Gentamicin loaded niosomes composed of Tween 60 , cholesterol, and dicetyl phosphate were the most effective in terms of prolongation of in vitro drug release [107].

5.5. Anti-Inflammatory Drugs. Nonsteroidal anti-inflammatory drugs (NSAIDs) loaded niosomes have been prepared by several groups. These drugs may cause adverse effects such as mucosal irritation. Topically applied NSAIDs loaded niosomes can substantially improve drug permeation. To investigate the potential application of the niosomes for delivery of anti-inflammatory agents, Marianecci et al. synthesized ammonium glycyrrhizinate (AG) loaded niosomes using several surfactants and cholesterol at various concentrations. Drug entrapment efficiency, anisotropy, cytotoxicity and skin tolerability, and some further analysis have been performed for characterization. The AG-loaded niosomes demonstrated no toxicity and good skin tolerability and were able to improve the anti-inflammatory activity in mice. Moreover, an enhancement of the anti-inflammatory activity of the niosome delivered drug was observed on chemically induced skin erythema in humans [7].

5.6. Antiviral Drugs. Niosomes have also demonstrated the capability to deliver various antiviral agents. Ruckmani and Sankar synthesized zidovudine, which is the first anti-HIV compound approved for clinical use, encapsulated niosomes, and examined their entrapment efficiency and as well as sustainability of release. The niosomes were formulated by combining the proportions of Tween, Span, and cholesterol. Niosomes composed Tween 80 entrapped large amounts of zidovudine and the addition of dicetyl phosphate enhanced drug release for a longer time [108]. The drug leakage from Tween 80 formulations stored at room temperature was significant compared to niosomes stored at $4^{\circ} \mathrm{C}$ for 90 days. Besides, the results of a pharmacokinetic study in rabbits also confirmed that Tween 80 formulations with dicetyl phosphate were cleared from the circulation within five hours [109].

\section{Recent Studies}

Over the past three decades, niosomes have been successfully used as a drug carriers to overcome some major biopharmaceutical problems such as insolubility, side effects, and 
TABLE 3: Recent studies on niosomes in drug delivery.

\begin{tabular}{|c|c|c|c|c|c|}
\hline Type of the drug & Name of the drug & Composition & Experimental model & Year & References \\
\hline $\begin{array}{l}\text { Angiotensin receptor } \\
\text { blockers }\end{array}$ & $\begin{array}{l}\text { Candesartan } \\
\text { cilexetil }\end{array}$ & $\begin{array}{l}\text { Span } 60 \text {, cholesterol, } \\
\text { dicetyl phosphate, } \\
\text { maltodextrin }\end{array}$ & $\begin{array}{l}\text { In vitro dissolution test for } \\
\text { proniosomal tablets, in vivo evaluation } \\
\text { of proniosomal tablets, } \\
\text { pharmacokinetic analysis }\end{array}$ & 2016 & [36] \\
\hline \multirow{2}{*}{ Anti-inflammatory } & Naproxen & $\begin{array}{l}\text { Tween } 80 \text {, Tween } 20 \text {, } \\
\text { cholesterol }\end{array}$ & $\begin{array}{l}\text { In vitro drug release study, } \\
\text { preformulation study }\end{array}$ & 2016 & [37] \\
\hline & Dexamethasone & Span 60, cholesterol & $\begin{array}{l}\text { Characterization of niosomes, in vitro } \\
\text { release studies, stability test }\end{array}$ & 2015 & [38] \\
\hline \multirow[b]{2}{*}{ Antibacterial } & Moxifloxacin & Tween 60 , cholesterol & $\begin{array}{l}\text { In vitro release studies, antimicrobial } \\
\text { activity }\end{array}$ & 2016 & [39] \\
\hline & Cefixime & $\begin{array}{l}\text { C-Glycoside derivative } \\
\text { surfactant, cholesterol }\end{array}$ & $\begin{array}{l}\text { In vitro release study, biocompatibility } \\
\text { and bioavailability studies using } \\
\text { experimental animals }\end{array}$ & 2016 & {$[21]$} \\
\hline \multirow[t]{2}{*}{ Anticancer } & Doxorubicin & $\begin{array}{l}\text { Span 60, cholesterol, } \\
\text { dicetyl phosphate, } \\
\text { N-lauryl glucosamine }\end{array}$ & $\begin{array}{l}\text { Optimization studies for formulation, } \\
\text { skin irritancy, histopathological } \\
\text { investigation of rat skin }\end{array}$ & 2016 & {$[35]$} \\
\hline & Paclitaxel & $\begin{array}{l}\text { Span } 40, \text { cholesterol, } \\
\text { dicetyl phosphate }\end{array}$ & $\begin{array}{l}\text { Formulation studies, Pharmacokinetic } \\
\text { and tissue distribution studies }\end{array}$ & 2015 & {$[6]$} \\
\hline Antiviral & Nevirapine & Tyloxapol, cholesterol & $\begin{array}{l}\text { Diffusion kinetics of drug, } \\
\text { microviscosity studies, in vitro release } \\
\text { study }\end{array}$ & 2015 & {$[8]$} \\
\hline $\mathrm{H}_{2}$ receptor antagonist & Famotidine & Span 60, cholesterol & $\begin{array}{l}\text { Kinetic analysis of drug-release } \\
\text { profiles, ex vivo permeability study }\end{array}$ & 2016 & {$[40]$} \\
\hline
\end{tabular}

poor chemical stability of drug molecules [110]. Table 3 summarizes the most recent applications of niosomes as drug delivery systems.

\section{Strengths and Limitations of Niosomes in Drug Delivery}

One of the most important strengths of niosomes compared with liposomes is their chemical stability. Niosomes are more stable against chemical degradation or oxidation and have long storage time compared to liposomes [51]. The surfactants which are used for niosomes preparation are biodegradable, biocompatible, and nonimmunogenic [83]. Handling and storage conditions of surfactants do not need any specifications. Moreover composition, size, lamellarity, stability, and surface charge of niosomes can be controlled by the type of preparation method, surfactant, cholesterol content, surface charge additives, and suspension concentration [66].

On the other hand niosomes show physical stability problems. During storage of dispersion niosomes are at risk of aggregation, fusion, drug leakage, or hydrolysis of encapsulated drugs. Furthermore the sterilization of niosomes needs much effort. Heat sterilization and membrane filtration are unsuitable for niosomes. Thus, these areas need further research to produce commercially niosomal preparations.

\section{Conclusion}

Niosomes are novel nano drug carriers to design effective drug delivery systems. They offer a great opportunity for loading hydrophilic, lipophilic drugs, or both drugs together.
Numbers of studies have been performed with different types of niosomes in delivery of the anticancer agents, antiinflammatory agents, anti-infective agents, and so forth. The relevant studies demonstrated that niosomes improve the stability of the entrapped drug, reduce the dose, and enable targeted delivery to a specific type of tissue. The structural properties and characteristics of the niosomes can be enhanced by using novel preparations, loading, and modification methods for particular routes of administration. Thus, niosomes present itself as promising tools in commercially available therapeutics.

\section{Competing Interests}

The authors declare that there are no competing interests regarding the publication of this paper.

\section{Acknowledgments}

Konrad Adenauer Foundation is acknowledged for the financial support to Didem Ag Seleci. The publication of this paper was funded by the Open Access Fund of the Leibniz Universität Hannover.

\section{References}

[1] M. Seleci, D. Ag Seleci, R. Joncyzk, F. Stahl, C. Blume, and T. Scheper, "Smart multifunctional nanoparticles in nanomedicine," BioNanoMaterials, vol. 17, no. 1-2, pp. 33-41, 2016.

[2] N. B. Mahale, P. D. Thakkar, R. G. Mali, D. R. Walunj, and S. R. Chaudhari, "Niosomes: novel sustained release nonionic 
stable vesicular systems-an overview," Advances in Colloid and Interface Science, vol. 183, pp. 46-54, 2012.

[3] L. Tavano, L. Gentile, C. Oliviero Rossi, and R. Muzzalupo, "Novel gel-niosomes formulations as multicomponent systems for transdermal drug delivery," Colloids and Surfaces B: Biointerfaces, vol. 110, pp. 281-288, 2013.

[4] K. B. Bini, D. Akhilesh, P. Prabhakara, and K. Jv, "Development and characterization of non-ionic surfactant vesicles (niosomes) for oral delivery of lornoxicam," International Journal of Drug Development and Research, vol. 4, no. 3, pp. 147-154, 2012.

[5] Q. Li, Z. Li, W. Zeng et al., "Proniosome-derived niosomes for tacrolimus topical ocular delivery: in vitro cornea permeation, ocular irritation, and in vivo anti-allograft rejection," European Journal of Pharmaceutical Sciences, vol. 62, pp. 115-123, 2014.

[6] Z. S. Bayindir, A. Beşikci, and N. Yüksel, "Paclitaxel-loaded niosomes for intravenous administration: pharmacokinetics and tissue distribution in rats," Turkish Journal of Medical Sciences, vol. 45, no. 6, pp. 1403-1412, 2015.

[7] C. Marianecci, F. Rinaldi, M. Mastriota et al., "Anti-inflammatory activity of novel ammonium glycyrrhizinate/niosomes delivery system: human and murine models," Journal of Controlled Release, vol. 164, no. 1, pp. 17-25, 2012.

[8] S. K. Mehta and N. Jindal, "Tyloxapol niosomes as prospective drug delivery module for antiretroviral drug nevirapine," AAPS PharmSciTech, vol. 16, no. 1, pp. 67-75, 2014.

[9] P. Arunothayanun, M.-S. Bernard, D. Q. M. Craig, I. F. Uchegbu, and A. T. Florence, "The effect of processing variables on the physical characteristics of non-ionic surfactant vesicles (niosomes) formed from a hexadecyl diglycerol ether," International Journal of Pharmaceutics, vol. 201, no. 1, pp. 7-14, 2000.

[10] A. Pardakhty, J. Varshosaz, and A. Rouholamini, "In vitro study of polyoxyethylene alkyl ether niosomes for delivery of insulin," International Journal of Pharmaceutics, vol. 328, no. 2, pp. 130141, 2007.

[11] M. Manconi, D. Valenti, C. Sinico, F. Lai, G. Loy, and A. M. Fadda, "Niosomes as carriers for tretinoin: II. Influence of vesicular incorporation on tretinoin photostability," International Journal of Pharmaceutics, vol. 260, no. 2, pp. 261-272, 2003.

[12] Z. S. Bayindir and N. Yuksel, "Characterization of niosomes prepared with various nonionic surfactants for paclitaxel oral delivery," Journal of Pharmaceutical Sciences, vol. 99, no. 4, pp. 2049-2060, 2010.

[13] D. Paolino, D. Cosco, R. Muzzalupo, E. Trapasso, N. Picci, and M. Fresta, "Innovative bola-surfactant niosomes as topical delivery systems of 5-fluorouracil for the treatment of skin cancer," International Journal of Pharmaceutics, vol. 353, no. 12, pp. 233-242, 2008.

[14] D. Paolino, R. Muzzalupo, A. Ricciardi, C. Celia, N. Picci, and M. Fresta, "In vitro and in vivo evaluation of Bola-surfactant containing niosomes for transdermal delivery," Biomedical Microdevices, vol. 9, no. 4, pp. 421-433, 2007.

[15] T. Yoshioka, B. Sternberg, and A. T. Florence, "Preparation and properties of vesicles (niosomes) of sorbitan monoesters (Span 20, 40,60 and 80) and a sorbitan triester (Span 85)," International Journal of Pharmaceutics, vol. 105, no. 1, pp. 1-6, 1994.

[16] V. C. Okore, A. A. Attama, K. C. Ofokansi, C. O. Esimone, and E. B. Onuigbo, "Formulation and evaluation of niosomes," Indian Journal of Pharmaceutical Sciences, vol. 73, no. 3, pp. 323-328, 2011.

[17] D. Akhilesh, K. B. Bini, and J. V. Kamath, "Review on span-60 based non-ionic surfactant vesicles (niosomes) as novel drug delivery," International Journal of Research in Pharmaceutical and Biomedical Sciences, vol. 3, pp. 6-12, 2012.

[18] C. P. Jain and S. P. Vyas, "Preparation and characterization of niosomes containing rifampicin for lung targeting," Journal of Microencapsulation, vol. 12, no. 4, pp. 401-407, 1995.

[19] S. Mandal, C. Banerjee, S. Ghosh, J. Kuchlyan, and N. Sarkar, "Modulation of the photophysical properties of curcumin in nonionic surfactant (Tween-20) forming micelles and niosomes: a comparative study of different microenvironments," The Journal of Physical Chemistry B, vol. 117, no. 23, pp. 69576968, 2013.

[20] L. Di Marzio, C. Marianecci, M. Petrone, F. Rinaldi, and M. Carafa, "Novel pH-sensitive non-ionic surfactant vesicles: comparison between Tween 21 and Tween 20," Colloids and Surfaces B: Biointerfaces, vol. 82, no. 1, pp. 18-24, 2011.

[21] M. Imran, M. R. Shah, F. Ullah et al., "Glycoside-based niosomal nanocarrier for enhanced in-vivo performance of Cefixime," International Journal of Pharmaceutics, vol. 505, no. 1-2, pp. 122132, 2016.

[22] M. Manconi, C. Sinico, D. Valenti, F. Lai, and A. M. Fadda, "Niosomes as carriers for tretinoin: III. A study into the in vitro cutaneous delivery of vesicle-incorporated tretinoin," International Journal of Pharmaceutics, vol. 311, no. 1-2, pp. 11-19, 2006.

[23] P. Bandyopadhyay and M. Johnson, "Fatty alcohols or fatty acids as niosomal hybrid carrier: effect on vesicle size, encapsulation efficiency and in vitro dye release," Colloids and Surfaces B: Biointerfaces, vol. 58, no. 1, pp. 68-71, 2007.

[24] L. Tavano, R. Muzzalupo, L. Mauro, M. Pellegrino, S. Andò, and N. Picci, "Transferrin-conjugated Pluronic niosomes as a new drug delivery system for anticancer therapy," Langmuir, vol. 29, no. 41, pp. 12638-12646, 2013.

[25] R. Muzzalupo, L. Tavano, R. Cassano, S. Trombino, T. Ferrarelli, and N. Picci, "A new approach for the evaluation of niosomes as effective transdermal drug delivery systems," European Journal of Pharmaceutics and Biopharmaceutics, vol. 79, no. 1, pp. 28-35, 2011.

[26] M. Bragagni, N. Mennini, S. Furlanetto, S. Orlandini, C. Ghelardini, and P. Mura, "Development and characterization of functionalized niosomes for brain targeting of dynorphin-B," European Journal of Pharmaceutics and Biopharmaceutics, vol. 87, no. 1, pp. 73-79, 2014.

[27] S. P. Vyas, R. P. Singh, S. Jain et al., "Non-ionic surfactant based vesicles (niosomes) for non-invasive topical genetic immunization against hepatitis B," International Journal of Pharmaceutics, vol. 296, no. 1-2, pp. 80-86, 2005.

[28] A. Sankhyan and P. Pawar, "Recent trends in niosome as vesicular drug delivery system," Journal of Applied Pharmaceutical Science, vol. 2, no. 6, pp. 20-32, 2012.

[29] V. B. Junyaprasert, V. Teeranachaideekul, and T. Supaperm, "Effect of charged and non-ionic membrane additives on physicochemical properties and stability of niosomes," AAPS PharmSciTech, vol. 9, no. 3, pp. 851-859, 2008.

[30] M. Bragagni, N. Mennini, C. Ghelardini, and P. Mura, "Development and characterization of niosomal formulations of doxorubicin aimed at brain targeting," Journal of Pharmacy and Pharmaceutical Sciences, vol. 15, no. 1, pp. 184-196, 2012.

[31] C. Dufes, F. Gaillard, I. F. Uchegbu, A. G. Schätzlein, J.-C. Olivier, and J.-M. Muller, "Glucose-targeted niosomes deliver vasoactive intestinal peptide (VIP) to the brain," International Journal of Pharmaceutics, vol. 285, no. 1-2, pp. 77-85, 2004. 
[32] L. Tavano, M. Vivacqua, V. Carito, R. Muzzalupo, M. C. Caroleo, and F. Nicoletta, "Doxorubicin loaded magneto-niosomes for targeted drug delivery," Colloids and Surfaces B: Biointerfaces, vol. 102, pp. 803-807, 2013.

[33] M. Hong, S. Zhu, Y. Jiang, G. Tang, and Y. Pei, "Efficient tumor targeting of hydroxycamptothecin loaded PEGylated niosomes modified with transferrin," Journal of Controlled Release, vol. 133, no. 2, pp. 96-102, 2009.

[34] C. Dufes, J.-M. Muller, W. Couet, J.-C. Olivier, I. F. Uchegbu, and A. G. Schätzlein, "Anticancer drug delivery with transferrin targeted polymeric chitosan vesicles," Pharmaceutical Research, vol. 21, no. 1, pp. 101-107, 2004.

[35] S. Pawar and P. Vavia, "Glucosamine anchored cancer targeted nano-vesicular drug delivery system of doxorubicin," Journal of Drug Targeting, vol. 24, no. 1, pp. 68-79, 2016.

[36] N. Yuksel, Z. S. Bayindir, E. Aksakal, and A. T. Ozcelikay, "In situ niosome forming maltodextrin proniosomes of candesartan cilexetil: in vitro and in vivo evaluations," International Journal of Biological Macromolecules, vol. 82, pp. 453-463, 2016.

[37] N. Shah, "Characterization, optimization and formulation of niosome containing naproxen," Journal of Biomedical and Pharmaceutical Research, vol. 5, no. 1, pp. 1-6, 2016.

[38] M. A. Mavaddati, F. Moztarzadeh, and F. Baghbani, "Effect of formulation and processing variables on dexamethasone entrapment and release of niosomes," Journal of Cluster Science, vol. 26, no. 6, pp. 2065-2078, 2015.

[39] S. Sohrabi, A. Haeri, A. Mahboubi, A. Mortazavi, and S. Dadashzadeh, "Chitosan gel-embedded moxifloxacin niosomes: an efficient antimicrobial hybrid system for burn infection," International Journal of Biological Macromolecules, vol. 85, pp. 625-633, 2016.

[40] V. J. Mokale, H. I. Patil, A. P. Patil, P. R. Shirude, and J. B. Naik, "Formulation and optimisation of famotidine proniosomes: an in vitro and ex vivo study," Journal of Experimental Nanoscience, vol. 11, no. 2, pp. 97-110, 2016.

[41] J. Jiao, "Polyoxyethylated nonionic surfactants and their applications in topical ocular drug delivery," Advanced Drug Delivery Reviews, vol. 60, no. 15, pp. 1663-1673, 2008.

[42] I. F. Uchegbu and A. T. Florence, "Non-ionic surfactant vesicles (niosomes): physical and pharmaceutical chemistry," Advances in Colloid and Interface Science, vol. 58, no. 1, pp. 1-55, 1995.

[43] A. Shahiwala and A. Misra, "Studies in topical application of niosomally entrapped nimesulide," Journal of Pharmacy and Pharmaceutical Sciences, vol. 5, no. 3, pp. 220-225, 2002.

[44] V. Sharma, S. Anandhakumar, and M. Sasidharan, "Selfdegrading niosomes for encapsulation of hydrophilic and hydrophobic drugs: an efficient carrier for cancer multi-drug delivery," Materials Science and Engineering: C, vol. 56, pp. 393400, 2015.

[45] G. Caracciolo, D. Pozzi, R. Caminiti et al., "Effect of hydration on the structure of solid-supported Niosomal membranes investigated by in situ energy dispersive X-ray diffraction," Chemical Physics Letters, vol. 462, no. 4-6, pp. 307-312, 2008.

[46] B. Nasseri, "Effect of cholesterol and temperature on the elastic properties of niosomal membranes," International Journal of Pharmaceutics, vol. 300, no. 1-2, pp. 95-101, 2005.

[47] C. Marianecci, L. Di Marzio, F. Rinaldi et al., "Niosomes from 80s to present: the state of the art," Advances in Colloid and Interface Science, vol. 205, pp. 187-206, 2014.

[48] G. P. Kumar and P. Rajeshwarrao, "Nonionic surfactant vesicular systems for effective drug delivery-an overview," Acta Pharmaceutica Sinica B, vol. 1, no. 4, pp. 208-219, 2011.
[49] S. Biswal, P. N. Murthy, J. Sahu, P. Sahoo, and F. Amir, "Vesicles of non-ionic surfactants (niosomes) and drug delivery potential," Internatinal Journal of Pharmaceutical Sciences and Nanotechnology, vol. 1, no. 1, pp. 1-8, 2008.

[50] J. N. Israelachvili, Intermolecular and Surface Forces, 1985, Academic Press, New York, NY, USA, 1985.

[51] I. F. Uchegbu and S. P. Vyas, "Non-ionic surfactant based vesicles (niosomes) in drug delivery," International Journal of Pharmaceutics, vol. 172, no. 1-2, pp. 33-70, 1998.

[52] R. A. Khalil and A.-H. A. Zarari, "Theoretical estimation of the critical packing parameter of amphiphilic self-assembled aggregates," Applied Surface Science, vol. 318, pp. 85-89, 2014.

[53] S. Moghassemi and A. Hadjizadeh, "Nano-niosomes as nanoscale drug delivery systems: an illustrated review," Journal of Controlled Release, vol. 185, no. 1, pp. 22-36, 2014.

[54] T. Liu, R. Guo, W. Hua, and J. Qiu, "Structure behaviors of hemoglobin in PEG 6000/Tween 80/Span 80/ $\mathrm{H}_{2}$ O niosome system," Colloids and Surfaces A: Physicochemical and Engineering Aspects, vol. 293, no. 1-3, pp. 255-261, 2007.

[55] S. Agarwal, V. Bakshi, P. Vitta, A. P. Raghuram, S. Pandey, and N. Udupa, "Effect of cholesterol content and surfactant HLB on vesicle properties of niosomes," Indian Journal of Pharmaceutical Sciences, vol. 66, no. 1, pp. 121-123, 2004.

[56] M. Mokhtar, O. A. Sammour, M. A. Hammad, and N. A. Megrab, "Effect of some formulation parameters on flurbiprofen encapsulation and release rates of niosomes prepared from proniosomes," International Journal of Pharmaceutics, vol. 361, no. 1-2, pp. 104-111, 2008.

[57] S. Bhaskaran and P. K. Lakshmi, "Comparative evaluation of niosome formulations prepared by different techniques," Acta Pharmaceutica Sciencia, vol. 51, no. 1, pp. 27-32, 2009.

[58] A. J. Baillie, A. T. Florence, L. R. Hume, G. T. Muirhead, and A. Rogerson, "The preparation and properties of niosomes non-ionic surfactant vesicles," The Journal of Pharmacy and Pharmacology, vol. 37, no. 12, pp. 863-868, 1985.

[59] A. Marwa, S. Omaima, E. L. G. Hanaa, and A.-S. Mohammed, "Preparation and in-vitro evaluation of diclofenac sodium niosomal formulations," International Journal of Pharmaceutical Sciences and Research, vol. 4, no. 5, pp. 1757-1765, 2013.

[60] A. Rogerson, J. Cummings, N. Willmott, and A. T. Florence, "The distribution of doxorubicin in mice following administration in niosomes," Journal of Pharmacy and Pharmacology, vol. 40, no. 5, pp. 337-342, 1988.

[61] S. Srinivas, Y. A. Kumar, A. Hemanth, and M. Anitha, "Preparation and evaluation of niosomes containing aceclofenac," Digest Journal of Nanomaterials and Biostructures, vol. 5, no. 1, pp. 249254, 2010.

[62] S. Moghassemi, E. Parnian, A. Hakamivala et al., "Uptake and transport of insulin across intestinal membrane model using trimethyl chitosan coated insulin niosomes," Materials Science and Engineering C, vol. 46, pp. 333-340, 2015.

[63] A. Budhiraja and G. Dhingra, "Development and characterization of a novel antiacne niosomal gel of rosmarinic acid," Drug Delivery, vol. 22, no. 6, pp. 723-730, 2015.

[64] H. Kiwada, H. Niimura, Y. Fujisaki, S. Yamada, and Y. Kato, "Application of synthetic alkyl glycoside vesicles as drug carriers. I. Preparation and physical properties," Chemical and Pharmaceutical Bulletin, vol. 33, no. 2, pp. 753-759, 1985.

[65] A. S. Zidan, Z. Rahman, and M. A. Khan, "Product and process understanding of a novel pediatric anti-HIV tenofovir niosomes with a high-pressure homogenizer," European Journal of Pharmaceutical Sciences, vol. 44, no. 1-2, pp. 93-102, 2011. 
[66] S. Verma, S. K. Singh, N. Syan, P. Mathur, and V. Valecha, "Nanoparticle vesicular systems: a versatile tool for drug delivery," Journal of Chemical and Pharmaceutical Research, vol. 2, no. 2, pp. 496-509, 2010.

[67] A. Manosroi, R. Chutoprapat, M. Abe, and J. Manosroi, "Characteristics of niosomes prepared by supercritical carbon dioxide $\left(\mathrm{scCO}_{2}\right)$ fluid," International Journal of Pharmaceutics, vol. 352, no. 1-2, pp. 248-255, 2008.

[68] A. Manosroi, W. Ruksiriwanich, M. Abe, H. Sakai, W. Manosroi, and J. Manosroi, "Biological activities of the rice bran extract and physical characteristics of its entrapment in niosomes by supercritical carbon dioxide fluid," The Journal of Supercritical Fluids, vol. 54, no. 2, pp. 137-144, 2010.

[69] V. R. Yasam, S. L. Jakki, J. Natarajan, and G. Kuppusamy, "A review on novel vesicular drug delivery: proniosomes," Drug Delivery, vol. 21, no. 4, pp. 243-249, 2014.

[70] L. D. Mayer, M. B. Bally, and P. R. Cullis, "Uptake of adriamycin into large unilamellar vesicles in response to a $\mathrm{pH}$ gradient," Biochimica et Biophysica Acta (BBA) - Biomembranes, vol. 857, no. 1, pp. 123-126, 1986.

[71] A. K. Verma and J. C. Bindal, "A vital role of niosomes on controlled and novel drug delivery," Indian Journal of Novel Drug Delivery, vol. 3, pp. 238-246, 2011.

[72] M. R. Mozafari, "A new technique for the preparation of nontoxic liposomes and nanoliposomes: the heating method," in Nanoliposomes: From Fundamentals to Recent Developments, pp. 91-98, Trafford Publishing, Oxford, UK, 2005.

[73] M. R. Mozafari, C. J. Reed, and C. Rostron, "Cytotoxicity evaluation of anionic nanoliposomes and nanolipoplexes prepared by the heating method without employing volatile solvents and detergents," Die Pharmazie, vol. 62, no. 3, pp. 205-209, 2007.

[74] H. Talsma, M. J. Van Steenbergen, J. C. H. Borchert, and D. J. A. Crommelin, "A novel technique for the one-step preparation of liposomes and nonionic surfactant vesicles without the use of organic solvents. Liposome formation in a continuous gas stream: the 'bubble' method," Journal of Pharmaceutical Sciences, vol. 83, no. 3, pp. 276-280, 1994.

[75] L. Tavano, R. Aiello, G. Ioele, N. Picci, and R. Muzzalupo, "Niosomes from glucuronic acid-based surfactant as new carriers for cancer therapy: preparation, characterization and biological properties," Colloids and Surfaces B: Biointerfaces, vol. 118, pp. 7-13, 2014.

[76] A. Priprem, K. Janpim, S. Nualkaew, and P. Mahakunakorn, "Topical niosome gel of Zingiber cassumunar Roxb. extract for anti-inflammatory activity enhanced skin permeation and stability of compound D," AAPS PharmSciTech, vol. 17, no. 3, pp. 631-639, 2016.

[77] W. Hua and T. Liu, "Preparation and properties of highly stable innocuous niosome in Span 80/PEG 400/ $\mathrm{H}_{2} \mathrm{O}$ system," Colloids and Surfaces A: Physicochemical and Engineering Aspects, vol. 302, no. 1, pp. 377-382, 2007.

[78] A. Manosroi, P. Wongtrakul, J. Manosroi et al., "Characterization of vesicles prepared with various non-ionic surfactants mixed with cholesterol," Colloids and Surfaces B: Biointerfaces, vol. 30, no. 1-2, pp. 129-138, 2003.

[79] D. Pozzi, R. Caminiti, C. Marianecci et al., "Effect of cholesterol on the formation and hydration behavior of solid-supported niosomal membranes," Langmuir, vol. 26, no. 4, pp. 2268-2273, 2010.

[80] D. Pando, G. Gutiérrez, J. Coca, and C. Pazos, "Preparation and characterization of niosomes containing resveratrol," Journal of Food Engineering, vol. 117, no. 2, pp. 227-234, 2013.
[81] M. Tabbakhian, S. Daneshamouz, N. Tavakoli, and M. R. Jaafari, "Influence of liposomes and niosomes on the in vitro permeation and skin retention of finasteride," Iranian Journal of Pharmaceutical Sciences, vol. 1, no. 3, pp. 119-130, 2005.

[82] S. K. Mehta and N. Jindal, "Formulation of Tyloxapol niosomes for encapsulation, stabilization and dissolution of antitubercular drugs," Colloids and Surfaces B: Biointerfaces, vol. 101, pp. 434-441, 2013.

[83] A. Y. Waddad, S. Abbad, F. Yu et al., "Formulation, characterization and pharmacokinetics of Morin hydrate niosomes prepared from various non-ionic surfactants," International Journal of Pharmaceutics, vol. 456, no. 2, pp. 446-458, 2013.

[84] Y. Hao, F. Zhao, N. Li, Y. Yang, and K. Li, "Studies on a high encapsulation of colchicine by a niosome system," International Journal of Pharmaceutics, vol. 244, no. 1-2, pp. 73-80, 2002.

[85] A. Dwivedi, A. Mazumder, L. du Plessis, J. L. du Preez, R. K. Haynes, and J. du Plessis, "In vitro anti-cancer effects of artemisone nano-vesicular formulations on melanoma cells," Nanomedicine: Nanotechnology, Biology, and Medicine, vol. 11, no. 8, pp. 2041-2050, 2015.

[86] R. P. Gude, M. G. Jadhav, S. G. A. Rao, and A. G. Jagtap, "Effects of niosomal cisplatin and combination of the same with theophylline and with activated macrophages in murine B16F10 melanoma model," Cancer Biotherapy and Radiopharmaceuticals, vol. 17, no. 2, pp. 183-192, 2002.

[87] D. Cosco, D. Paolino, R. Muzzalupo et al., "Novel PEG-coated niosomes based on bola-surfactant as drug carriers for 5fluorouracil," Biomedical Microdevices, vol. 11, no. 5, pp. 11151125, 2009.

[88] W. Han, S. Wang, R. Liang et al., "Non-ionic surfactant vesicles simultaneously enhance antitumor activity and reduce the toxicity of cantharidin," International Journal of Nanomedicine, vol. 8, pp. 2187-2196, 2013.

[89] D. S. Shaker, M. A. Shaker, and M. S. Hanafy, "Cellular uptake, cytotoxicity and in-vivo evaluation of Tamoxifen citrate loaded niosomes," International Journal of Pharmaceutics, vol. 493, no. 1-2, pp. 285-294, 2015.

[90] I. F. Uchegbu, J. A. Double, L. R. Kelland, J. A. Turton, and A. T. Florence, "The activity of doxorubicin niosomes against an ovarian cancer cell line and three in vivo mouse tumour models," Journal of Drug Targeting, vol. 3, no. 5, pp. 399-409, 1996.

[91] D. J. Kerr, A. Rogerson, G. J. Morrison, A. T. Florence, and S. B. Kaye, "Antitumour activity and pharmacokinetics of niosome encapsulated adriamycin in monolayer, spheroid and xenograft," British Journal of Cancer, vol. 58, no. 4, pp. 432-436, 1988.

[92] S. Y. Gaikwad, A. G. Jagtap, A. D. Ingle, S. G. A. Ra, and R. P. Gude, "Antimetastatic efficacy of niosomal pentoxifylline and its combination with activated macrophages in murine B16F10 melanoma model," Cancer Biotherapy \& Radiopharmaceuticals, vol. 15, no. 6, pp. 605-615, 2000.

[93] M. Kong, H. Park, C. Feng, L. Hou, X. Cheng, and X. Chen, "Construction of hyaluronic acid noisome as functional transdermal nanocarrier for tumor therapy," Carbohydrate Polymers, vol. 94, no. 1, pp. 634-641, 2013.

[94] A. Narang and R. Mahato, Targeted Delivery of Small and Macromolecular Drugs, CRC Press, 2010.

[95] D. Ag, R. Bongartz, L. E. Dogan et al., "Biofunctional quantum dots as fluorescence probe for cell-specific targeting," Colloids and Surfaces B: Biointerfaces, vol. 114, pp. 96-103, 2014. 
[96] M. Seleci, D. A. Seleci, M. Ciftci et al., "Nanostructured amphiphilic star-hyperbranched block copolymers for drug delivery," Langmuir, vol. 31, no. 15, pp. 4542-4551, 2015.

[97] S. Gadde, "Multi-drug delivery nanocarriers for combination therapy," MedChemComm, vol. 6, no. 11, pp. 1916-1929, 2015.

[98] B. Al-Lazikani, U. Banerji, and P. Workman, "Combinatorial drug therapy for cancer in the post-genomic era," Nature Biotechnology, vol. 30, no. 7, pp. 679-692, 2012.

[99] G. Pasut, F. Greco, A. Mero et al., "Polymer-drug conjugates for combination anticancer therapy: investigating the mechanism of action," Journal of Medicinal Chemistry, vol. 52, no. 20, pp. 6499-6502, 2009.

[100] Y. D. Livney and Y. G. Assaraf, "Rationally designed nanovehicles to overcome cancer chemoresistance," Advanced Drug Delivery Reviews, vol. 65, no. 13-14, pp. 1716-1730, 2013.

[101] C. Sarisozen, I. Vural, T. Levchenko, A. A. Hincal, and V. P. Torchilin, "PEG-PE-based micelles co-loaded with paclitaxel and cyclosporine A or loaded with paclitaxel and targeted by anticancer antibody overcome drug resistance in cancer cells," Drug Delivery, vol. 19, no. 4, pp. 169-176, 2012.

[102] M. Thakkar and S. Brijesh, "Opportunities and challenges for niosomes as drug delivery systems," Current Drug Delivery, vol. 13, pp. 1-15, 2016.

[103] L. Tavano, R. Muzzalupo, N. Picci, and B. De Cindio, "Coencapsulation of antioxidants into niosomal carriers: gastrointestinal release studies for nutraceutical applications," Colloids and Surfaces B: Biointerfaces, vol. 114, pp. 82-88, 2014.

[104] C. Marianecci, F. Rinaldi, L. D. Marzio, A. Ciogli, S. Esposito, and M. Carafa, "Polysorbate 20 vesicles as multi-drug carriers: in vitro preliminary evaluations," Letters in Drug Design and Discovery, vol. 10, no. 3, pp. 212-218, 2013.

[105] K. Begum, A. F. Khan, H. K. Hana, J. Sheak, and R. U. Jalil, "Rifampicin niosome: preparations, characterizations and antibacterial activity against staphylococcus aureus and staphylococcus epidermidis isolated from acne," Dhaka University Journal of Pharmaceutical Sciences, vol. 14, no. 1, pp. 117-123, 2015.

[106] V. Akbari, D. Abedi, A. Pardakhty, and H. Sadeghi-Aliabadi, "Release studies on ciprofloxacin loaded non-ionic surfactant vesicles," Avicenna Journal of Medical Biotechnology, vol. 7, no. 2, pp. 69-75, 2015.

[107] G. Abdelbary and N. El-Gendy, "Niosome-encapsulated gentamicin for ophthalmic controlled delivery," AAPS PharmSciTech, vol. 9, no. 3, pp. 740-747, 2008.

[108] K. Ruckmani and V. Sankar, "Formulation and optimization of zidovudine niosomes," AAPS PharmSciTech, vol. 11, no. 3, pp. 1119-1127, 2010.

[109] K. Ruckmani, V. Sankar, and M. Sivakumar, "Tissue distribution, pharmacokinetics and stability studies of zidovudine delivered by niosomes and proniosomes," Journal of Biomedical Nanotechnology, vol. 6, no. 1, pp. 43-51, 2010.

[110] H. Abdelkader, A. W. G. Alani, and R. G. Alany, "Recent advances in non-ionic surfactant vesicles (niosomes): selfassembly, fabrication, characterization, drug delivery applications and limitations," Drug Delivery, vol. 21, no. 2, pp. 87-100, 2014. 

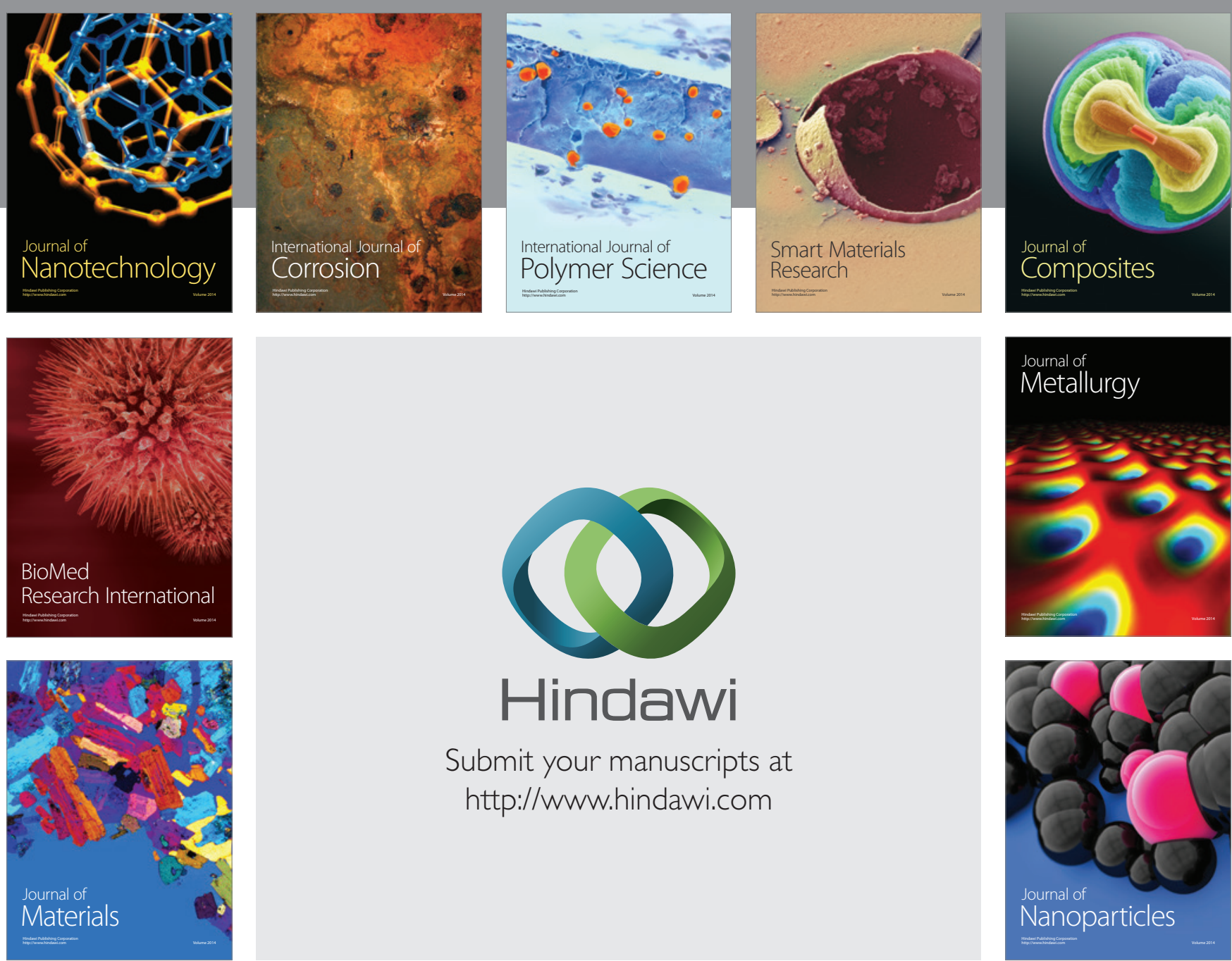

\section{Hindawi}

Submit your manuscripts at

http://www.hindawi.com

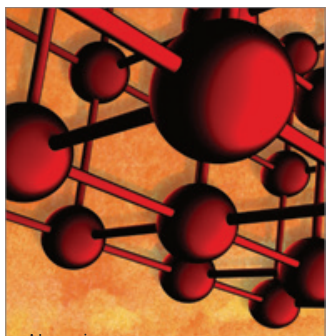

Materials Science and Engineering
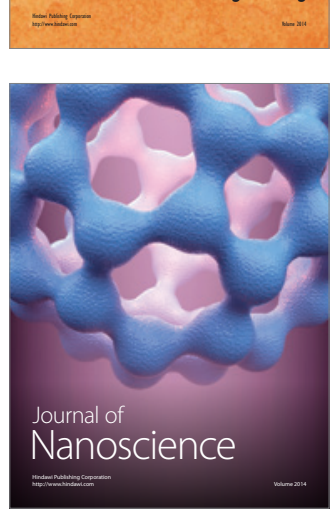
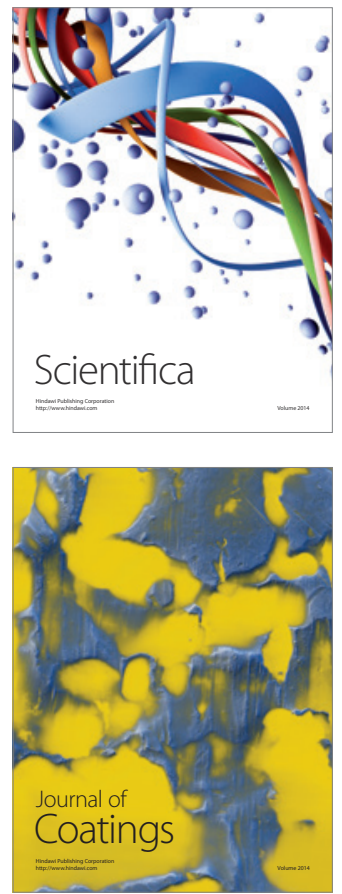
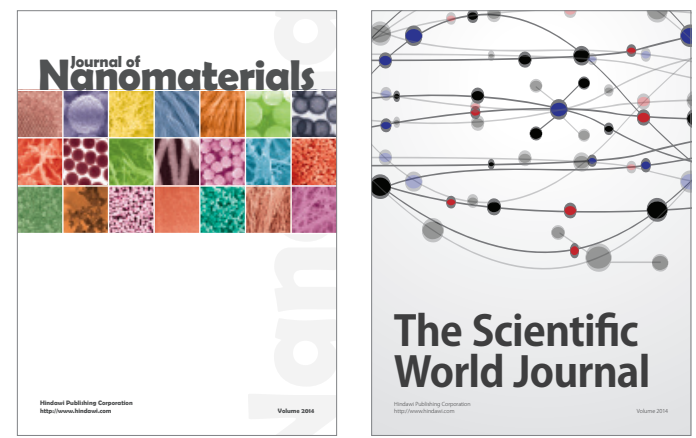

The Scientific World Journal
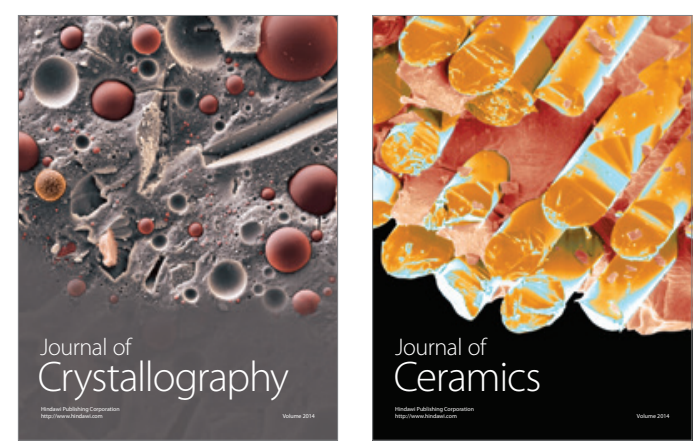
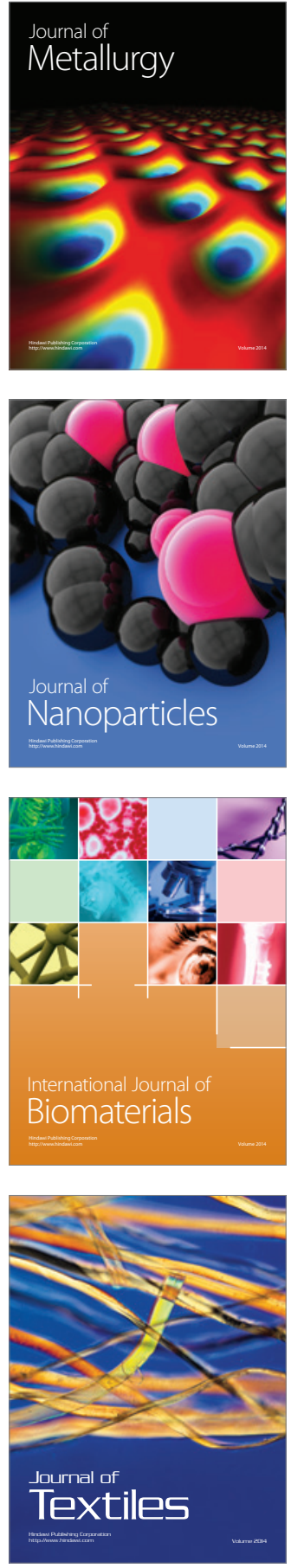